\title{
ON THE CORRELATION BETWEEN THE VOLUMES OF THE TYPICAL POISSON-VORONOI CELL AND THE TYPICAL STIENEN SPHERE
}

\author{
VIKTOR OLSBO, ${ }^{*}$ Chalmers University of Technology and Göteborg University
}

\begin{abstract}
In this paper we consider a tessellation $\mathcal{V}$ generated by a homogeneous Poisson process $\Phi$ in $\mathbb{R}^{d}$ and, furthermore, the random set of spheres with centres being the points in $\Phi$ and having radii equal to half the distance to their closest neighbouring point in $\Phi$. In $\mathbb{R}^{d}$ we give an integral formula for the correlation between the volume of the typical cell and the volume of the sphere in the typical cell, and we also show that this correlation is strictly positive. Furthermore, on the real line we give an analytical expression for the correlation, and in the plane and in space we give simplified integral formulae. Numerical values for the correlation for $d=2, \ldots, 7$ are also given.

Keywords: Correlation; Poisson process; Robbins' formula; Stienen model; typical cell; Voronoi tessellation

2000 Mathematics Subject Classification: Primary 60D05
\end{abstract}

Secondary 60G55

\section{Introduction}

Let $\Phi$ be a homogeneous Poisson process in $\mathbb{R}^{d}$ with intensity $\lambda \in(0, \infty)$ and let $\mathcal{V}$ denote the tessellation generated by $\Phi$. This means that, for each point $x \in \Phi$, we let $\mathcal{V}_{x}$ be the cell that has the point $\boldsymbol{x}$ as its nucleus (or generator), i.e. $\mathcal{V}_{\boldsymbol{x}}=\left\{\boldsymbol{y} \in \mathbb{R}^{d}:\|\boldsymbol{y}-\boldsymbol{x}\| \leq\|\boldsymbol{y}-\boldsymbol{z}\|, \boldsymbol{z} \in \Phi\right\}$, where $\|\cdot\|$ denotes the Euclidean norm, and then $\mathcal{V}=\left\{\mathcal{V}_{x}: x \in \Phi\right\}$. The tessellation $\mathcal{V}$ is known as the Poisson-Voronoi tessellation and was introduced by Meijering [5]. For a more in-depth view of Poisson-Voronoi tessellations, the reader is referred to [6] and [7]. Calka [1], [2] investigated the size and the form of Voronoi cells in the plane. Hug et al. [4] investigated the shape of large Voronoi cells.

In order to describe the statistical properties of $\mathcal{V}$ it is useful to introduce the typical cell. Following the approach introduced in [6], we let $\Gamma$ denote the set of polytopes in $\mathbb{R}^{d}$ equipped with a 'suitable' $\sigma$-field $\mathcal{A}$. For a set $A \in \mathcal{A}$, we define the following distribution:

$$
Q(A)=\frac{1}{\lambda v_{d}(B)} \mathrm{E}\left[\sum_{x \in \Phi \cap B} \mathbf{1}\left(\mathcal{V}_{\boldsymbol{x}}-\boldsymbol{x} \in A\right)\right]
$$

for an arbitrary Borel set $B$ such that $0<v_{d}(B)<\infty$. Here, $v_{d}$ denotes the Lebesgue measure. The typical Voronoi cell is defined as the random polytope $\mathcal{V}_{\mathrm{t}}$ on $(\Gamma, \mathcal{A})$ having distribution $Q$. It is known (see, e.g. [6]) that $\mathcal{V}_{\mathrm{t}}$ has the same distribution as $\mathcal{V}_{\mathrm{o}}$, where $\mathcal{V}_{\mathrm{o}}$ denotes the cell whose nucleus is the origin. This means that the distribution $\mathrm{P}$ of $\Phi$ is given by the Palm distribution at the origin. To emphasize this we use the notation $\Phi_{0}$. On the real line $v_{1}\left(\mathcal{V}_{0}\right) \sim \Gamma(2,2 \lambda)$

Received 19 January 2007; revision received 6 September 2007.

* Postal address: Mathematical Sciences, Chalmers University of Technology and Göteborg University, SE-412 96 Göteborg, Sweden. Email address: vikol@ chalmers.se 

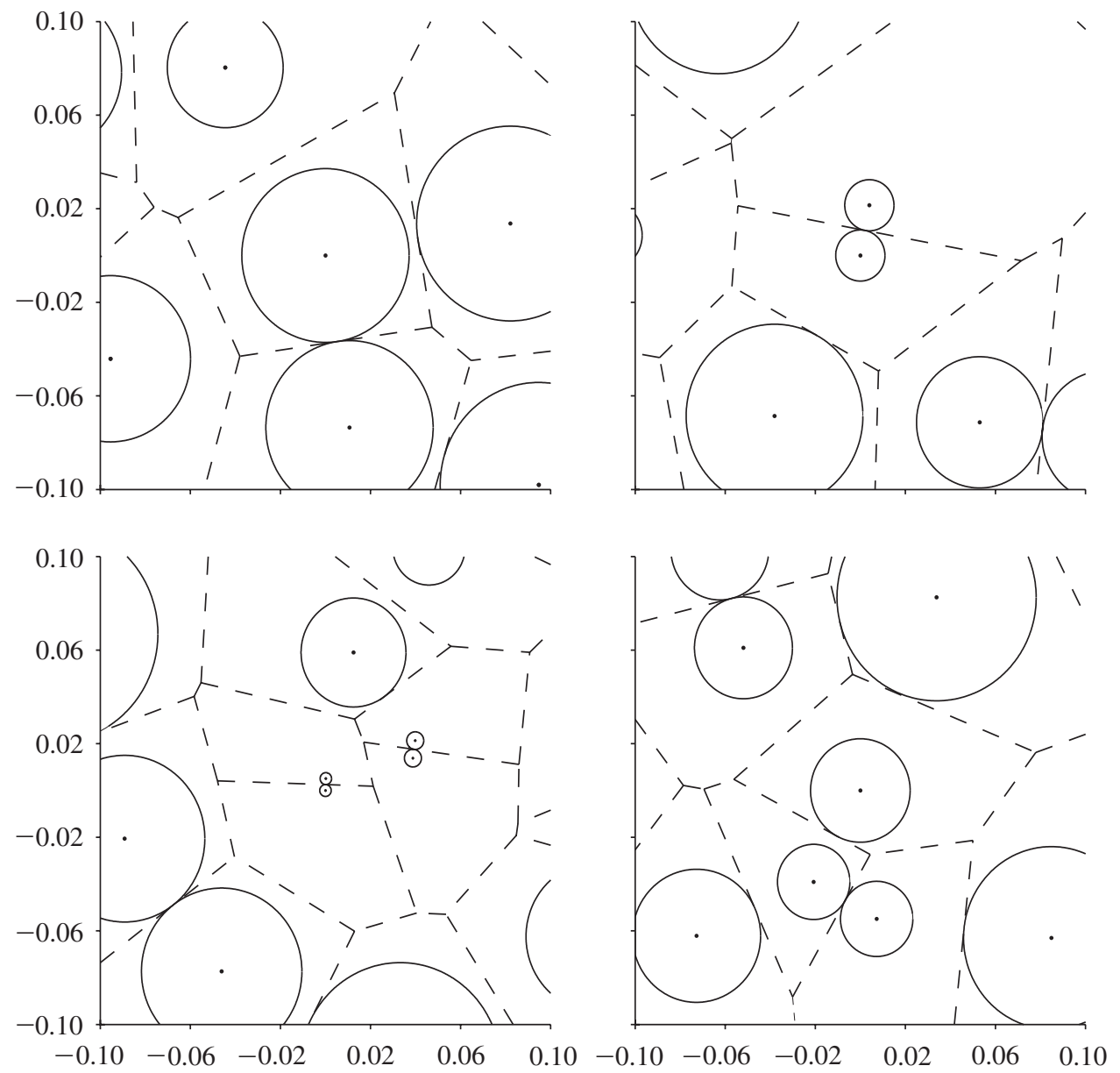

FIGURE 1: Examples of realizations of $\mathcal{V}_{\mathrm{O}}$ and $\varsigma_{0}$.

and, for $\mathbb{R}^{2}$, Calka [2] gave an expression for both the density of the number of neighbours and the conditional distribution function of the area of the typical cell, conditioned on the number of neighbours. For $d \geq 3$, the distribution of the volume of the typical cell is unknown.

Around each point $\boldsymbol{x} \in \Phi$ we place a sphere with diameter equal to the distance to the closest neighbour of $\boldsymbol{x}$. The random closed set made up of the union of these spheres is known as the Stienen model and was introduced by Stienen [11], motivated by an application in materials science. Conditioning on $\Phi$ with a point at the origin, we call the sphere having the origin as its centre the typical Stienen sphere (or the typical sphere) and denote it by $\S_{\mathrm{o}}$. Distributional properties of the Stienen model were studied by Schlater and Stoyan [10]. Although the distributions of the volumes of the typical sphere and the typical cell (at least for $d=2$ ) are known, the correlation between them is not available in the literature. At first it seems obvious that this correlation must be positive, but examining the selected realizations in Figure 1 suggests that the answer is not this obvious as, for example, two neighbouring points lying close together often produce small spheres and large Voronoi cells. 


\section{Main results}

In this section we state and discuss the main results while the proofs are postponed to Section 3. In Proposition 2.1, below, we give a closed-form expression for the correlation between $v_{1}\left(\mathcal{V}_{\mathrm{o}}\right)$ and $v_{1}\left(\wp_{\mathrm{o}}\right)$. In Theorem 2.1, below, we give an integral formula for $\operatorname{corr}\left(v_{d}\left(\mathcal{V}_{\mathrm{o}}\right), v_{d}\left(\wp_{\mathrm{o}}\right)\right)$ in arbitrary dimensions, $d \geq 2$.

Proposition 2.1. On the real line it holds that

$$
\operatorname{corr}\left(v_{1}\left(\mathcal{V}_{\mathrm{o}}\right), v_{1}\left(\wp_{\mathrm{o}}\right)\right)=\frac{1}{\sqrt{2}}
$$

The real line is the only case where we have been able to obtain an analytical expression for $\operatorname{corr}\left(v_{d}\left(\mathcal{V}_{\mathrm{o}}\right), v_{d}\left(\wp_{\mathrm{o}}\right)\right)$. An integral formula for $\operatorname{corr}\left(v_{d}\left(\mathcal{V}_{\mathrm{o}}\right), v_{d}\left(\wp_{\mathrm{o}}\right)\right)$ in arbitrary dimensions, $d \geq 2$, is given in Theorem 2.1, below. Lemma 3.1 in Section 3 gives an integral formula that holds for any $d \geq 1$.

Theorem 2.1. For $d \geq 2$, the correlation between the volume of $\mathcal{V}_{\mathrm{o}}$ and the volume of $\varsigma_{\mathrm{o}}$ is given by

$$
\begin{aligned}
& \operatorname{corr}\left(v_{d}\left(\mathcal{V}_{\mathrm{o}}\right), v_{d}\left(\wp_{\mathrm{o}}\right)\right) \\
& =\frac{\sigma_{d}^{2} \int_{0}^{\infty} \int_{0}^{2 v} u^{d-1} v^{d-1} \mathrm{e}^{-b_{d-1} W_{d}(u, v)} \mathrm{d} u \mathrm{~d} v-\left(2^{d}-1\right) / 2^{d}}{\sqrt{(d-1)\left(b_{d} / b_{d-1}\right) \int_{0}^{\pi} \int_{0}^{\infty}\left(\sin ^{d-2}(\alpha) R^{d-1} / S_{d}(R, \alpha)^{2}\right) \mathrm{d} R \mathrm{~d} \alpha-1}},
\end{aligned}
$$

where

$$
W_{d}(u, v)=u^{d} \int_{\arccos (u / 2 v)}^{\pi} \sin ^{d} t \mathrm{~d} t+v^{d} \int_{\arccos \left(2 v^{2}-u^{2} / 2 v^{2}\right)}^{\pi} \sin ^{d} t \mathrm{~d} t
$$

and

$$
S_{d}(R, \alpha)=R^{d} \int_{\alpha}^{\pi} \sin ^{d} t \mathrm{~d} t+\left(R^{2}+1-2 R \cos \alpha\right)^{d / 2} \int_{T(R, \alpha)}^{\pi} \sin ^{d} t \mathrm{~d} t,
$$

with

$$
T(R, \alpha)=\arccos \left(\frac{1-R \cos \alpha}{\sqrt{R^{2}+1-2 R \cos \alpha}}\right) .
$$

Here, $\sigma_{d}$ and $b_{d}$ denote the surface area and the volume of the $d$-dimensional unit sphere, respectively.

The function $b_{d-1} W_{d}(u, v)$ can be interpreted as the volume of the union of two $d$-dimensional spheres with radii $u$ and $v$ and their centres a distance $v$ apart. Furthermore, $b_{d-1} S_{d}(R, \alpha)$ can be interpreted as the volume of two spheres, with their centres a unit distance apart, where one has radius $R$ and the other has radius $\sqrt{R^{2}+1-2 R \cos \alpha}$. For any $d \geq 2$, the integrals defining $S_{d}(R, \alpha)$ and $W_{d}(u, v)$ can be evaluated in terms of elementary functions. This means that, for example, in the plane and in space it is straightforward to use (2.1) to obtain numerical approximations for the correlation between $v_{d}\left(\mathcal{V}_{\mathrm{o}}\right)$ and $v_{d}\left(\delta_{\mathrm{o}}\right)$.

Corollary 2.1. In the plane and in space the following two assertions hold.

$$
\operatorname{corr}\left(\nu_{2}\left(\mathcal{V}_{\mathrm{o}}\right), \nu_{2}\left(\wp_{\mathrm{o}}\right)\right)=\frac{4 \pi^{2} \tau_{2}-3 / 4}{\sqrt{(\pi / 2) I_{1}-1}} \approx 0.7051
$$


where

$$
\tau_{1}=\int_{0}^{\pi} \int_{0}^{\infty} \frac{R}{S_{2}(R, \alpha)^{2}} \mathrm{~d} R \mathrm{~d} \alpha
$$

and

$$
\tau_{2}=\int_{0}^{\infty} \int_{0}^{2 v} u v \mathrm{e}^{-2 W_{2}(u, v)} \mathrm{d} u \mathrm{~d} v
$$

with

$$
\begin{aligned}
& S_{2}(R, \alpha)=\frac{1}{2}\left\{\left(1+R^{2}-2 R \cos \alpha\right)\left(\pi-\arccos \left(\frac{1-R \cos \alpha}{\sqrt{1+R^{2}-2 R \cos \alpha}}\right)\right)\right. \\
& \left.+R^{2}(\pi-\alpha)+R \sin \alpha\right\}
\end{aligned}
$$

and

$$
W_{2}(u, v)=\frac{u^{2}}{2}\left(\pi-\arccos \frac{u}{2 v}\right)+\frac{v^{2}}{2}\left(\pi-\arccos \left(1-\frac{u^{2}}{2 v^{2}}\right)\right)+\frac{u}{4} \sqrt{4 v^{2}-u^{2}} .
$$

(ii)

$$
\operatorname{corr}\left(v_{3}\left(\mathcal{V}_{\mathrm{o}}\right), v_{3}\left(\wp_{\mathrm{o}}\right)\right)=\frac{16 \pi^{2} g_{2}-7 / 8}{\sqrt{(8 / 3) g_{1}-1}} \approx 0.6778
$$

where

$$
g_{1}=\int_{0}^{\pi} \int_{0}^{\infty} \frac{R^{2} \sin \alpha}{S_{3}(R, \alpha)^{2}} \mathrm{~d} R \mathrm{~d} \alpha
$$

and

$$
g_{2}=\int_{0}^{\infty} \int_{0}^{2 v} u^{2} v^{2} \exp \left(-\frac{\pi\left(3 u^{4}+8 u^{3} v+16 v^{4}\right)}{12 v}\right) \mathrm{d} u \mathrm{~d} v
$$

with

$$
S_{3}(R, \alpha)=\frac{1}{6}\left(4+9 R^{2}+4 R^{3}-12 R \cos \alpha+4\left(1+R^{2}-2 R \cos \alpha\right)^{3 / 2}+3 R^{2} \cos 2 \alpha\right) .
$$

Table 1 displays numerical estimates of $\operatorname{corr}\left(v_{d}\left(\mathcal{V}_{\mathrm{o}}\right), v_{d}\left(\wp_{\mathrm{o}}\right)\right)$ for $d=1, \ldots, 7$. The numerical results were obtained using the NIntegrate routine in MATHEMATICA ${ }^{\circledR}$. As can be seen from Table 1, the correlation seems to decrease as the dimension increases. Using the bounds in (2.4), below, it follows that $\operatorname{corr}\left(v_{d}\left(\mathcal{V}_{\mathrm{o}}\right), v_{d}\left(\delta_{\mathrm{o}}\right)\right) \rightarrow 0$ as $d \rightarrow \infty$.

TABLE 1: Numerical estimates of $\operatorname{corr}\left(v_{d}\left(\mathcal{V}_{\mathrm{o}}\right), v_{d}\left(\wp_{\mathrm{o}}\right)\right)$ for $d=1, \ldots, 7$.

\begin{tabular}{cc}
\hline$d$ & $\operatorname{corr}\left(v_{d}\left(\mathcal{V}_{\mathrm{o}}\right), v_{d}\left(\ell_{\mathrm{o}}\right)\right)$ \\
\hline 1 & 0.707107 \\
2 & 0.705143 \\
3 & 0.677790 \\
4 & 0.649534 \\
5 & 0.623393 \\
6 & 0.599667 \\
7 & 0.578145 \\
\hline
\end{tabular}


It was questioned above whether $\operatorname{corr}\left(v_{d}\left(\mathcal{V}_{\mathrm{o}}\right), v_{d}\left(\wp_{\mathrm{o}}\right)\right)$ is positive or not. As seen, we cannot find a closed form for the correlation between $v_{d}\left(\mathcal{V}_{\mathrm{o}}\right)$ and $v_{d}\left(\wp_{\mathrm{o}}\right)$ in arbitrary dimensions, but we can give a lower bound which shows that it is strictly positive, a result which is in accordance with intuition. Furthermore, we give bounds for the covariance between $v_{d}\left(\mathcal{V}_{\mathrm{o}}\right)$ and $v_{d}\left(\delta_{\mathrm{o}}\right)$.

Proposition 2.2. For any dimension, it holds that

(i)

$$
\frac{1}{2^{2 d}\left(1+2^{d}\right) \lambda^{2}} \leq \operatorname{cov}\left(v_{d}\left(\mathcal{V}_{\mathrm{o}}\right), v_{d}\left(\wp_{\mathrm{o}}\right)\right) \leq \frac{1}{2^{d} \lambda^{2}},
$$

(ii)

$$
\operatorname{corr}\left(v_{d}\left(\mathcal{V}_{\mathrm{o}}\right), v_{d}\left(f_{\mathrm{o}}\right)\right) \geq \frac{1}{2^{d}\left(1+2^{d}\right)} .
$$

A result which lies a little outside the main focus of this article is the following proposition, which gives bounds on the second moment of the volume of the typical cell in arbitrary dimensions.

Proposition 2.3. It holds that

$$
\frac{1}{\lambda^{2}} \leq \mathrm{E}\left[v_{d}\left(\mathcal{V}_{\mathrm{o}}\right)^{2}\right] \leq \frac{2}{\lambda^{2}} .
$$

Remark 2.1. The lower bound in Proposition 2.3 is elementary since $E\left[v_{d}\left(\mathcal{V}_{0}\right)\right]=1 / \lambda$; see (3.3), below. Also note that, for $d=1$, we have $\mathrm{E}\left[v_{1}\left(\mathcal{V}_{\mathrm{o}}\right)^{2}\right]=3 / 2 \lambda^{2}$.

\section{Proofs}

A result, given in [8] and [9], that will be central in the proofs states that

$$
\mathrm{E}\left[v_{d}(X)^{n}\right]=\int_{\mathbb{R}^{d}} \cdots \int_{\mathbb{R}^{d}} \mathrm{P}\left(\boldsymbol{x}_{1}, \ldots, \boldsymbol{x}_{n} \in X\right) \mathrm{d} \boldsymbol{x}_{1} \cdots \mathrm{d} \boldsymbol{x}_{n}
$$

for any random closed set $X$.

\subsection{Moments of the typical cell}

Equation (3.1) implies that

$$
\begin{aligned}
\mathrm{E}\left[v_{d}\left(\mathcal{V}_{\mathrm{o}}\right)^{n}\right] & =\int_{\mathbb{R}^{d}} \cdots \int_{\mathbb{R}^{d}} \mathrm{P}\left(\boldsymbol{x}_{1}, \ldots, \boldsymbol{x}_{n} \in \mathcal{V}_{\mathrm{o}}\right) \mathrm{d} \boldsymbol{x}_{1} \cdots \mathrm{d} \boldsymbol{x}_{n} \\
& =\int_{\mathbb{R}^{d}} \cdots \int_{\mathbb{R}^{d}} \mathrm{e}^{-\lambda U_{d}\left(\boldsymbol{x}_{1}, \ldots, \boldsymbol{x}_{n}\right)} \mathrm{d} \boldsymbol{x}_{1} \cdots \mathrm{d} \boldsymbol{x}_{n},
\end{aligned}
$$

where $U_{d}\left(\boldsymbol{x}_{1}, \ldots, \boldsymbol{x}_{n}\right)$ is the volume of the union of $n$ spheres in $\mathbb{R}^{d}$, centred at $\boldsymbol{x}_{1}, \ldots, \boldsymbol{x}_{n}$ and having radii $\left\|x_{1}\right\|, \ldots,\left\|x_{n}\right\|$, respectively. The property that

$$
\mathrm{P}\left(\boldsymbol{x}_{1}, \ldots, \boldsymbol{x}_{n} \in \mathcal{V}_{\mathrm{o}}\right)=\mathrm{e}^{-\lambda U\left(\boldsymbol{x}_{1}, \ldots, \boldsymbol{x}_{n}\right)}
$$

follows from the fact that the points $\boldsymbol{x}_{1}, \ldots, \boldsymbol{x}_{n}$ lie inside the typical cell if and only if

$$
\Phi_{\mathrm{o}}^{!} \cap \bigcup_{i=1, \ldots, n} B_{d}\left(\boldsymbol{x}_{i},\left\|\boldsymbol{x}_{i}\right\|\right)=\varnothing
$$


where $\Phi_{\mathrm{o}}^{!}$denotes $\Phi_{\mathrm{o}} \backslash\{\mathrm{o}\}$ and $B_{d}(\boldsymbol{x}, r)=\left\{\boldsymbol{y} \in \mathbb{R}^{d}:\|\boldsymbol{x}-\boldsymbol{y}\| \leq r\right\}$. It is well known that

$$
\mathrm{E}\left[v_{d}\left(\mathcal{V}_{\mathrm{o}}\right)\right]=\frac{1}{\lambda},
$$

which is straightforward to obtain from (3.2). This result was initially given in [3]. Unfortunately it is the only moment known in analytical form, except for the special case in which $d=1$, where all moments are known. On the real line, $\mathcal{V}_{\mathrm{o}}$ is an interval. The distance from the origin to the left-hand endpoint of $\mathcal{V}_{\mathrm{o}}$ and the distance from the origin to the right-hand endpoint of $\mathcal{V}_{\mathrm{o}}$ are two independent $\operatorname{Exp}(2 \lambda)$-distributed random variables. This means that $v_{1}\left(\mathcal{V}_{\mathrm{o}}\right) \sim \Gamma(2,2 \lambda)$, which implies that

$$
\mathrm{E}\left[v_{1}\left(\mathcal{V}_{\mathrm{o}}\right)^{n}\right]=\frac{(n+1) !}{(2 \lambda)^{n}} .
$$

\subsection{Distribution of the typical sphere}

Let $R_{\mathrm{O}}$ denote the radius of a typical sphere in the Stienen model, i.e. the sphere that has the origin as its centre. We have $\mathrm{P}\left(R_{\mathrm{O}}>r\right)=\mathrm{e}^{-\lambda 2^{d} b_{d} r^{d}}$, as $R_{\mathrm{O}}$ is greater than $r$ if and only if $\Phi_{\mathrm{o}}^{!} \cap B_{d}(\mathrm{o}, 2 r)=\varnothing$. This means that

$$
\mathrm{P}\left(v_{d}\left(S_{\mathrm{o}}\right)>s\right)=\mathrm{P}\left(b_{d} R_{\mathrm{o}}^{d}>s\right)=\mathrm{P}\left(R_{\mathrm{o}}>\left(\frac{s}{b_{d}}\right)^{1 / d}\right)=\mathrm{e}^{-\lambda 2^{d} s},
$$

i.e. $v_{d}\left(S_{\mathrm{o}}\right) \sim \operatorname{Exp}\left(2^{d} \lambda\right)$; hence,

$$
\mathrm{E}\left[v_{d}\left(S_{\mathrm{o}}\right)^{n}\right]=\frac{n !}{\left(2^{d} \lambda\right)^{n}} .
$$

\subsection{Proofs of Proposition 2.1 and Theorem 2.1}

In this section we give the proofs of Theorem 2.1 and Proposition 2.1. To prove these results we will use the following lemma, which gives an integral formula for the correlation between $v_{d}\left(\mathcal{V}_{\mathrm{o}}\right)$ and $v_{d}\left(\wp_{\mathrm{o}}\right)$ for any $d$.

Lemma 3.1. The correlation between the volume of $\mathcal{V}_{\mathrm{o}}$ and the volume of $\S_{\mathrm{o}}$ is given by

$$
\operatorname{corr}\left(v_{d}\left(\mathcal{V}_{\mathrm{o}}\right), v_{d}\left(\wp_{\mathrm{o}}\right)\right)=\frac{\left(1-2^{d}\right) / 2^{d}+\iint_{\left\|\boldsymbol{x}_{1}\right\| \leq 2\left\|\boldsymbol{x}_{2}\right\|} \mathrm{e}^{-V_{d}\left(\boldsymbol{x}_{1}, \boldsymbol{x}_{2}\right)} \mathrm{d} \boldsymbol{x}_{1} \mathrm{~d} \boldsymbol{x}_{2}}{\sqrt{\int_{\mathbb{R}^{d}} \int_{\mathbb{R}^{d}} \mathrm{e}^{-U_{d}\left(\boldsymbol{x}_{1}, \boldsymbol{x}_{2}\right)} \mathrm{d} \boldsymbol{x}_{1} \mathrm{~d} \boldsymbol{x}_{2}-1}},
$$

where $V_{d}\left(\boldsymbol{x}_{1}, \boldsymbol{x}_{2}\right)$ is the volume of the union of two spheres in $\mathbb{R}^{d}$, centred at the origin and $\boldsymbol{x}_{2}$, with radii $\left\|\boldsymbol{x}_{1}\right\|$ and $\left\|\boldsymbol{x}_{2}\right\|$, respectively.

Proof. Define $\tilde{\mathcal{V}}_{\mathrm{o}}$ as the part of $\mathcal{V}_{\mathrm{o}}$ that lies outside $\varsigma_{\mathrm{o}}$, i.e. $\tilde{\mathcal{V}}_{\mathrm{o}}=\mathcal{V}_{\mathrm{o}} \backslash \S_{\mathrm{o}}$. Now, to obtain the correlation between $v_{d}\left(\mathcal{V}_{\mathrm{o}}\right)$ and $\nu_{d}\left(\delta_{\mathrm{o}}\right)$ we first compute the covariance. We have

$$
\begin{aligned}
\operatorname{var}\left(v_{d}\left(\tilde{\mathcal{V}}_{\mathrm{o}}\right)\right) & =\operatorname{var}\left(v_{d}\left(\mathcal{V}_{\mathrm{o}}\right)-v_{d}\left(\wp_{\mathrm{o}}\right)\right) \\
& =\operatorname{var}\left(v_{d}\left(\mathcal{V}_{\mathrm{o}}\right)\right)+\operatorname{var}\left(v_{d}\left(\wp_{\mathrm{o}}\right)\right)-2 \operatorname{cov}\left(v_{d}\left(\mathcal{V}_{\mathrm{o}}\right), v_{d}\left(\wp_{\mathrm{o}}\right)\right),
\end{aligned}
$$

which implies that

$$
\operatorname{cov}\left(v_{d}\left(\mathcal{V}_{\mathrm{o}}\right), v_{d}\left(\S_{\mathrm{o}}\right)\right)=\frac{1}{2}\left(\operatorname{var}\left(v_{d}\left(\mathcal{V}_{\mathrm{o}}\right)\right)+\operatorname{var}\left(v_{d}\left(\S_{\mathrm{o}}\right)\right)-\operatorname{var}\left(v_{d}\left(\tilde{\mathcal{V}}_{\mathrm{o}}\right)\right)\right) .
$$


Now, from (3.2), (3.3), and (3.5) it follows that

$$
\operatorname{var}\left(v_{d}\left(\mathcal{V}_{\mathrm{o}}\right)\right)=\int_{\mathbb{R}^{d}} \int_{\mathbb{R}^{d}} \mathrm{e}^{-\lambda U_{d}\left(\boldsymbol{x}_{1}, \boldsymbol{x}_{2}\right)} \mathrm{d} \boldsymbol{x}_{1} \mathrm{~d} \boldsymbol{x}_{2}-\frac{1}{\lambda^{2}}
$$

and

$$
\operatorname{var}\left(v_{d}\left(\delta_{0}\right)\right)=\frac{1}{\left(2^{d} \lambda\right)^{2}} .
$$

It remains to find $\operatorname{var}\left(v_{d}\left(\tilde{\mathcal{V}}_{\mathrm{o}}\right)\right)$. The expectation is given by

$$
\mathrm{E}\left[v_{d}\left(\tilde{\mathcal{V}}_{\mathrm{o}}\right)\right]=\mathrm{E}\left[v_{d}\left(\mathcal{V}_{\mathrm{o}}\right)-v_{d}\left(\wp_{\mathrm{o}}\right)\right]=\frac{2^{d}-1}{2^{d} \lambda} .
$$

This is what we would expect since the volume fraction of the Stienen model is equal to $2^{-d}$. We will use (3.1) to find the second moment of $v_{d}\left(\tilde{\mathcal{V}}_{\mathrm{o}}\right)$; then we will need to compute $\mathrm{P}\left(\boldsymbol{x}_{1}, \boldsymbol{x}_{2} \in \tilde{\mathcal{V}}_{\mathrm{o}}\right)$. Now, for $\left\|\boldsymbol{x}_{1}\right\| \leq\left\|\boldsymbol{x}_{2}\right\|$, we have

$$
\mathrm{P}\left(x_{1}, x_{2} \in \tilde{\mathcal{V}}_{\mathrm{o}}\right)=\mathrm{P}\left(R_{\mathrm{o}}<\left\|x_{1}\right\|, x_{1}, x_{2} \in \mathcal{V}_{\mathrm{o}}\right)
$$

and

$$
\mathrm{P}\left(x_{1}, x_{2} \in \mathcal{V}_{\mathrm{o}}\right)=\mathrm{P}\left(R_{\mathrm{o}}<\left\|x_{1}\right\|, x_{1}, x_{2} \in \mathcal{V}_{\mathrm{o}}\right)+\mathrm{P}\left(R_{\mathrm{o}} \geq\left\|x_{1}\right\|, x_{1}, x_{2} \in \mathcal{V}_{\mathrm{o}}\right) .
$$

Furthermore, $x_{1}$ and $x_{2}$ lie in $\mathcal{V}_{\mathrm{o}}$ and $R_{\mathrm{O}} \geq\left\|\boldsymbol{x}_{1}\right\|$ if and only if there are no points of $\Phi_{\mathrm{o}}^{!}$in $B_{d}\left(\mathrm{o}, 2\left\|x_{1}\right\|\right) \cup B_{d}\left(x_{2},\left\|x_{2}\right\|\right)$ and, therefore,

$$
\mathrm{P}\left(R_{\mathrm{o}} \geq\left\|\boldsymbol{x}_{1}\right\|, \boldsymbol{x}_{1}, \boldsymbol{x}_{2} \in \mathcal{V}_{\mathrm{o}}\right)=\mathrm{e}^{-\lambda V_{d}\left(x_{1}, x_{2}, 2\right)},
$$

where

$$
V_{d}\left(\boldsymbol{x}_{1}, \boldsymbol{x}_{2}, 2\right)=v_{d}\left(B_{d}\left(\mathrm{o}, 2\left\|\boldsymbol{x}_{1}\right\|\right) \cup B_{d}\left(\boldsymbol{x}_{2},\left\|\boldsymbol{x}_{2}\right\|\right)\right) .
$$

Using (3.1), the above means that

$$
\mathrm{E}\left[v_{d}\left(\tilde{\mathcal{V}}_{\mathrm{o}}\right)^{2}\right]=\int_{\mathbb{R}^{d}} \int_{\mathbb{R}^{d}} \mathrm{e}^{-\lambda U_{d}\left(\boldsymbol{x}_{1}, \boldsymbol{x}_{2}\right)} \mathrm{d} \boldsymbol{x}_{1} \mathrm{~d} \boldsymbol{x}_{2}-2 \iint_{\left\|\boldsymbol{x}_{1}\right\| \leq\left\|\boldsymbol{x}_{2}\right\|} \mathrm{e}^{-\lambda V_{d}\left(\boldsymbol{x}_{1}, \boldsymbol{x}_{2}, 2\right)} \mathrm{d} \boldsymbol{x}_{1} \mathrm{~d} \boldsymbol{x}_{2} .
$$

Using (3.2), (3.5), (3.9), and (3.10), (3.7) now becomes

$$
\begin{aligned}
\operatorname{cov}\left(v_{d}\left(\mathcal{V}_{\mathrm{o}}\right), v_{d}\left(\delta_{\mathrm{o}}\right)\right)= & \frac{1}{2} \int_{\mathbb{R}^{d}} \int_{\mathbb{R}^{d}} \mathrm{e}^{-\lambda U_{d}\left(\boldsymbol{x}_{1}, \boldsymbol{x}_{2}\right)} \mathrm{d} \boldsymbol{x}_{1} \mathrm{~d} \boldsymbol{x}_{2}-\frac{1}{2 \lambda^{2}}+\frac{1}{2\left(2^{d} \lambda\right)^{2}} \\
& -\frac{1}{2} \int_{\mathbb{R}^{d}} \int_{\mathbb{R}^{d}} \mathrm{e}^{-\lambda U_{d}\left(\boldsymbol{x}_{1}, \boldsymbol{x}_{2}\right)} \mathrm{d} \boldsymbol{x}_{1} \mathrm{~d} \boldsymbol{x}_{2} \\
& +\iint_{\left\|\boldsymbol{x}_{1}\right\| \leq\left\|\boldsymbol{x}_{2}\right\|} \mathrm{e}^{-\lambda V_{d}\left(\boldsymbol{x}_{1}, \boldsymbol{x}_{2}, 2\right)} \mathrm{d} \boldsymbol{x}_{1} \mathrm{~d} \boldsymbol{x}_{2}+\frac{\left(2^{d}-1\right)^{2}}{2\left(2^{d} \lambda\right)^{2}} \\
= & \frac{1-2^{d}}{\left(2^{d} \lambda\right)^{2}}+\iint_{\left\|\boldsymbol{x}_{1}\right\| \leq\left\|\boldsymbol{x}_{2}\right\|} \mathrm{e}^{-\lambda V_{d}\left(\boldsymbol{x}_{1}, \boldsymbol{x}_{2}, 2\right)} \mathrm{d} \boldsymbol{x}_{1} \mathrm{~d} \boldsymbol{x}_{2} .
\end{aligned}
$$

Finally, (3.2), (3.5), and (3.7) imply that

$$
\operatorname{corr}\left(v_{d}\left(\mathcal{V}_{\mathrm{o}}\right), v_{d}\left(\delta_{\mathrm{o}}\right)\right)=\frac{\left(1-2^{d}\right) /\left(2^{d} \lambda\right)^{2}+\iint_{\left\|\boldsymbol{x}_{1}\right\| \leq\left\|\boldsymbol{x}_{2}\right\|} \mathrm{e}^{-\lambda V_{d}\left(\boldsymbol{x}_{1}, \boldsymbol{x}_{2}, 2\right)} \mathrm{d} \boldsymbol{x}_{1} \mathrm{~d} \boldsymbol{x}_{2}}{\left(1 / 2^{d} \lambda\right) \sqrt{\int_{\mathbb{R}^{d}} \int_{\mathbb{R}^{d}} \mathrm{e}^{-\lambda U_{d}\left(\boldsymbol{x}_{1}, \boldsymbol{x}_{2}\right)} \mathrm{d} \boldsymbol{x}_{1} \mathrm{~d} \boldsymbol{x}_{2}-1 / \lambda^{2}}} .
$$


The change of variables $\left(x_{i 1}, \ldots, x_{i d}\right) \mapsto\left(y_{i 1} \lambda^{-1 / d}, \ldots, y_{i d} \lambda^{-1 / d}\right), i=1,2$, has the Jacobian $\lambda^{-1}$, giving a factor of $\lambda^{-2}$ outside each double integral. We also have

$$
\lambda U_{d}\left(\boldsymbol{x}_{1}, \boldsymbol{x}_{2}\right)=U_{d}\left(\boldsymbol{y}_{1}, \boldsymbol{y}_{2}\right) \quad \text { and } \quad \lambda V_{d}\left(\boldsymbol{x}_{1}, \boldsymbol{x}_{2}, 2\right)=V_{d}\left(\boldsymbol{y}_{1}, \boldsymbol{y}_{2}, 2\right) .
$$

Furthermore, the change of variables

$$
\left(x_{11}, \ldots, x_{1 d}\right) \mapsto\left(z_{11} 2^{-1}, \ldots, z_{1 d} 2^{-1}\right)
$$

has the Jacobian $2^{-d}$ and it further holds that $V_{d}\left(\boldsymbol{x}_{1}, \boldsymbol{x}_{2}, 2\right)=V_{d}\left(z_{1}, \boldsymbol{x}_{2}, 1\right)=V_{d}\left(z_{1}, \boldsymbol{x}_{2}\right)$. The integration limits become $\left\|z_{1}\right\| \leq 2\left\|x_{2}\right\|$. This completes the proof.

For $d=1$, the integrals in (3.6) can be calculated explicitly, but instead we will use the fact that $v_{1}\left(\delta_{0}\right)$ and $v_{1}\left(\tilde{\mathcal{V}}_{\mathrm{o}}\right)$ are independent, which follows from the basic properties of the Poisson process. The above directly yields

$$
\operatorname{corr}\left(v_{1}\left(\mathcal{V}_{\mathrm{o}}\right), v_{1}\left(\ell_{\mathrm{o}}\right)\right)=\sqrt{\frac{\operatorname{var}\left(v_{1}\left(\mathcal{S}_{\mathrm{o}}\right)\right)}{\operatorname{var}\left(\nu_{1}\left(\mathcal{V}_{\mathrm{o}}\right)\right)}}=\frac{1}{\sqrt{2}} .
$$

The last equality follows from (3.4) and (3.8). For $d \geq 2$, consider the following. Let $\alpha=$ $\angle\left(\mathrm{o}, \boldsymbol{x}_{1}, \boldsymbol{x}_{2}\right), r=\left\|\boldsymbol{x}_{1}-\boldsymbol{x}_{2}\right\|$, and $R=\left\|\boldsymbol{x}_{1}\right\| /\left\|\boldsymbol{x}_{1}-\boldsymbol{x}_{2}\right\|$. Then, by using the results in [3], it follows that

$$
\begin{aligned}
& \int_{\mathbb{R}^{d}} \int_{\mathbb{R}^{d}} \mathrm{e}^{-U_{d}\left(\boldsymbol{x}_{1}, \boldsymbol{x}_{2}\right)} \mathrm{d} \boldsymbol{x}_{1} \mathrm{~d} \boldsymbol{x}_{2} \\
& \quad=d(d-1) b_{d} b_{d-1} \int_{0}^{\infty} \int_{0}^{\pi} \int_{0}^{\infty} \sin ^{d-2}(\alpha) \mathrm{e}^{-b_{d-1} S_{d}(R, \alpha) r^{d}} r^{2 d-1} R^{d-1} \mathrm{~d} R \mathrm{~d} \alpha \mathrm{d} r
\end{aligned}
$$

where $S_{d}(R, \alpha)$ is as defined in (2.3). By integrating over $r$, we obtain the double integral of the denominator in (2.1). Furthermore, (2.2) holds since $B_{d}\left(\mathrm{o},\left\|x_{1}\right\|\right) \cup B_{d}\left(x_{2},\left\|x_{2}\right\|\right)=$ $s\left(\left\|x_{1}\right\|, t_{1}\right) \cup \delta\left(\left\|x_{2}\right\|, t_{2}\right)$, where $\delta\left(\left\|x_{1}\right\|, t_{1}\right)$ and $\delta\left(\left\|x_{2}\right\|, t_{2}\right)$ are two disjoint, truncated $d$-dimensional spheres of radii $\left\|\boldsymbol{x}_{1}\right\|$ and $\left\|\boldsymbol{x}_{2}\right\|$, respectively, truncated at distances

$$
t_{1}=\frac{\left\|x_{1}\right\|^{2}}{2\left\|x_{2}\right\|} \quad \text { and } \quad t_{2}=\frac{2\left\|x_{2}\right\|^{2}-\left\|x_{1}\right\|^{2}}{2\left\|x_{2}\right\|}
$$

from their respective centres. Geometric considerations and straightforward calculations show that the volume of each of these spheres is equal to $b_{d-1}$ times the corresponding term in (2.2). Changing to polar coordinates yields the expression in the denominator of (2.1).

\subsection{Proofs of Propositions 2.2 and 2.3}

We will use (3.6) to obtain bounds on the covariance. All we require are bounds on $\iint_{\left\|x_{1}\right\| \leq 2\left\|x_{2}\right\|} \mathrm{e}^{-V_{d}\left(\boldsymbol{x}_{1}, \boldsymbol{x}_{2}\right)} \mathrm{d} \boldsymbol{x}_{1} \mathrm{~d} \boldsymbol{x}_{2}$. As $V_{d}\left(\boldsymbol{x}_{1}, \boldsymbol{x}_{2}\right) \leq b_{d}\left(\left\|\boldsymbol{x}_{1}\right\|^{d}+\left\|\boldsymbol{x}_{2}\right\|^{d}\right)$, we have

$$
\begin{aligned}
\iint_{\left\|x_{1}\right\| \leq 2\left\|x_{2}\right\|} \mathrm{e}^{-V_{d}\left(\boldsymbol{x}_{1}, \boldsymbol{x}_{2}\right)} \mathrm{d} \boldsymbol{x}_{1} \mathrm{~d} \boldsymbol{x}_{2} & \geq \iint_{\left\|x_{1}\right\| \leq 2\left\|\boldsymbol{x}_{2}\right\|} \mathrm{e}^{-b_{d}\left(\left\|\boldsymbol{x}_{1}\right\|^{d}+\left\|\boldsymbol{x}_{2}\right\|^{d}\right)} \mathrm{d} \boldsymbol{x}_{1} \mathrm{~d} \boldsymbol{x}_{2} \\
& =\frac{1}{1+2^{d}} .
\end{aligned}
$$


To obtain an upper bound we first use the fact that

$$
\begin{aligned}
\iint_{\left\|x_{1}\right\| \leq 2\left\|x_{2}\right\|} \mathrm{e}^{-V_{d}\left(\boldsymbol{x}_{1}, \boldsymbol{x}_{2}\right)} \mathrm{d} \boldsymbol{x}_{1} \mathrm{~d} \boldsymbol{x}_{2}= & \iint_{\left\|x_{1}\right\| \leq\left\|x_{2}\right\|} \mathrm{e}^{-V_{d}\left(\boldsymbol{x}_{1}, \boldsymbol{x}_{2}\right)} \mathrm{d} \boldsymbol{x}_{1} \mathrm{~d} \boldsymbol{x}_{2} \\
& +\iint_{\left\|x_{2}\right\| \leq\left\|\boldsymbol{x}_{1}\right\| \leq 2\left\|\boldsymbol{x}_{2}\right\|} \mathrm{e}^{-V_{d}\left(\boldsymbol{x}_{1}, \boldsymbol{x}_{2}\right)} \mathrm{d} \boldsymbol{x}_{1} \mathrm{~d} \boldsymbol{x}_{2}
\end{aligned}
$$

and, furthermore, since $V_{d}\left(\boldsymbol{x}_{1}, \boldsymbol{x}_{2}\right) \geq b_{d}\left\|\boldsymbol{x}_{2}\right\|^{d}$, the facts that

$$
\iint_{\left\|x_{1}\right\| \leq\left\|\boldsymbol{x}_{2}\right\|} \mathrm{e}^{-V_{d}\left(\boldsymbol{x}_{1}, \boldsymbol{x}_{2}\right)} \mathrm{d} \boldsymbol{x}_{1} \mathrm{~d} \boldsymbol{x}_{2} \leq \frac{2 \pi^{d / 2}}{\Gamma(d / 2)} \int_{\mathbb{R}^{d}} \mathrm{e}^{-b_{d}\left\|\boldsymbol{x}_{2}\right\|^{d}} \int_{0}^{\left\|\boldsymbol{x}_{2}\right\|} r^{d-1} \mathrm{~d} r \mathrm{~d} \boldsymbol{x}_{2}=1
$$

and

$$
\begin{aligned}
\iint_{\left\|x_{2}\right\| \leq\left\|x_{1}\right\| \leq 2\left\|x_{2}\right\|} \mathrm{e}^{-V_{d}\left(x_{1}, \boldsymbol{x}_{2}\right)} \mathrm{d} \boldsymbol{x}_{1} \mathrm{~d} \boldsymbol{x}_{2} & \leq \frac{2 \pi^{d / 2}}{\Gamma(d / 2)} \int_{\mathbb{R}^{d}} \int_{\left\|x_{2}\right\|}^{2\left\|x_{2}\right\|} r^{d-1} \mathrm{e}^{-b_{d} r^{d}} \mathrm{~d} r \mathrm{~d} \boldsymbol{x}_{2} \\
& =1-\frac{1}{2^{d}} .
\end{aligned}
$$

Using the above, together with (3.11), proves Proposition 2.2(i). Furthermore, by symmetry, we have

$$
\int_{\mathbb{R}^{d}} \int_{\mathbb{R}^{d}} \mathrm{e}^{-U_{d}\left(\boldsymbol{x}_{1}, \boldsymbol{x}_{2}\right)} \mathrm{d} \boldsymbol{x}_{1} \mathrm{~d} \boldsymbol{x}_{2}=2 \iint_{\left\|\boldsymbol{x}_{1}\right\| \leq\left\|\boldsymbol{x}_{2}\right\|} \mathrm{e}^{-U_{d}\left(\boldsymbol{x}_{1}, \boldsymbol{x}_{2}\right)} \mathrm{d} \boldsymbol{x}_{1} \mathrm{~d} \boldsymbol{x}_{2} \leq 2,
$$

where we have used the fact that $U_{d}\left(\boldsymbol{x}_{1}, \boldsymbol{x}_{2}\right) \geq b_{d}\left\|\boldsymbol{x}_{2}\right\|^{d}$. The lower bound in (3.12) and the upper bound in (3.13) yield Proposition 2.2(ii). Finally, as

$$
\int_{\mathbb{R}^{d}} \int_{\mathbb{R}^{d}} \mathrm{e}^{-\lambda U_{d}\left(\boldsymbol{x}_{1}, \boldsymbol{x}_{2}\right)} \mathrm{d} \boldsymbol{x}_{1} \mathrm{~d} \boldsymbol{x}_{2}=\mathrm{E}\left[v_{d}\left(\mathcal{V}_{\mathrm{o}}\right)^{2}\right],
$$

(3.13) together with Remark 2.1 yield Proposition 2.3.

\section{Acknowledgements}

This research has been funded by the Swedish Foundation for Strategic Research and the Swedish Research Council, through the Stochastic Centre and GMMC, respectively. The author is very grateful for useful comments and suggestions from Aila Särkkä, Mats Kvarnström, and Tommy Norberg. The author also acknowledges the comments from the editor and an anonymous referee.

\section{References}

[1] Calka, P. (2002). The distribution of the smallest disks containing the Poisson-Voronoi typical cell and the Crofton cell in the plane. Adv. Appl. Prob. 34, 702-717.

[2] Calka, P. (2003). Precise formulae for the distributions of the principal geometric characteristics of the typical cells of a two-dimensional Poisson-Voronoi tessellation and a Poisson line process. Adv. Appl. Prob. 35, 551-562.

[3] Gilbert, E. N. (1962). Random subdivisions of space into crystals. Ann. Math. Statist. 33, 958-972.

[4] Hug, D., Reitzner, M. And Schneider, R. (2002). Large Poisson-Voronoi cells and Crofton cells. Adv. Appl. Prob. 34, 702-717.

[5] Meijering, J. L. (1953). Interface area, edge length and number of vertices in crystal aggregates with random nucleation. Philips Res. Rep. 8, 270-290.

[6] MøLler, J. (1994). Lectures on Random Voronoi Tessellations. Springer, New York. 
[7] Okabe, A., Boots, B., Sugihara, K. and Chiu, S. N. (2000). Spatial Tessellations, Concepts and Applications of Voronoi Diagrams, 2nd edn. John Wiley, Chichester.

[8] Robisns, H. E. (1944). On the measure of a random set. I. Ann. Math. Statist. 15, 70-74.

[9] Robbins, H. E. (1945). On the measure of a random set. II. Ann. Math. Statist. 16, 342-347.

[10] Schlater, M. and Stoyan, D. (1997). The covariance of the Stienen model. In Advances in Theory and Applications of Random Sets, ed. D. Jeulin, World Scientific, Singapore, pp. 157-174.

[11] Stienen, H. (1982). Die Vergröberung von Karbiden in reinen Eisen-Kohlenstoff Stählen. Doctoral Thesis, RWTH Aachen. 